\title{
Two-Storage Fuzzy Inventory Model with Time Dependent Demand and Holding Cost under Acceptable Delay in Payment
}

\section{Boina Anil Kumar ${ }^{a}$, Susanta Kumar Paikray ${ }^{a}$ and Umakanta Misra $^{b}$}

${ }^{a}$ Department of Mathematics

Veer Surendra Sai University of Technology, Burla, 768018 Odisha, India.

${ }^{b}$ Department of Mathematics

National Institute of Science and Technology, Palurhills, Berhampur, 761008

Odisha, India.

E-mail(corresp.): skpaikray_math@vssut.ac.in

E-mail: anilkumarboina@gmail.com

E-mail: umakanta_misra@yahoo.com

Received July 30, 2019; revised May 18, 2020; accepted May 18, 2020

\begin{abstract}
If we observe a real business market, the demand for items in each cycle is not in the same pattern, that is, for specific business cycle it may increase, stable or decrease (for instance, cool drinks from end stage of the summer to winter; the demand goes on decreasing, and from the end of winter to peak time of summer; the demand goes on increasing). Also, if the supplier permits for delay in payment, retailer wishes to buy more goods, and for which the retailer may need extra storage (in terms of a rented warehouse). Moreover, the retailer has always wished to sell the items before they expire and accordingly order is placed. Mostly the parameters in a real world inventory model are imprecise. Thus, in the proposed study an inventory model having decreasing time dependent demand pattern with variable holding cost for TwoStorage facility under acceptable delay in payment has been developed. Mathematical model of the problem and its solution procedure is discussed for both crisp and fuzzy environment in order to obtain the optimal replenishment time and cost. Also, numerical examples are discussed to validate the study. Finally, sensitivity analysis is also studied to describe the fluctuating scenario of associated parameters.
\end{abstract}

Keywords: inventory model, two-storage facility, variable holding cost, time dependent demand, delay in payment, fuzzy inventory model, defuzzification, graded mean integration representation.

AMS Subject Classification: 90B05; 03E72.

Copyright (c) 2020 The Author(s). Published by VGTU Press

This is an Open Access article distributed under the terms of the Creative Commons Attribution License (http://creativecommons.org/licenses/by/4.0/), which permits unrestricted use, distribution, and reproduction in any medium, provided the original author and source are credited. 


\section{Introduction}

The main aim of any business firm is to get more profit by expanding their business, creating goodwill in the market, and increasing their brand value. For which they consider many aspects, one of them is the inventory management system which plays an important role in any business affairs. An inventory management depends on different parameters such as demand, deterioration, holding cost, shortages, backlogs, inflation and trade credit, etc.

In the present global scenario, most of the suppliers offer price discount or trade credit financing. This may motivate retailers to purchase items more than that of fixed capacity of existing warehouse. To store these excess items retailer may hire a new warehouse on rental basis. Also, some retailers purchase a huge amount of items that cannot be accommodated in existing storage when a new product having very high demand launched into the market or when seasonal products arrives into the market. In fact, retailers of many products (for instance, apparels, footwear, jewellery, cosmetics, two wheelers, interior decorative items and marbles, etc.) use their primary warehouse at a suitable place in a busy market and that has been decorated with basic facilities to attract the customers for enhancement of their sales. At the same time, they used to rent or own a secondary warehouse to store the items in some other place to avoid heavy rent or maintenance cost.

Trade credit financing also plays an important role in inventory management. Allowing the trade credit finance by the supplier to retailers, supplier gets more quantity orders from existing customers and also attracts the new customers. As a result, sales of the supplier increases and in-hand stock level decreases quickly. Thus, the trade credit policy is helpful for the supplier to get more profit by decreasing the inventory cost. On the other hand, by availing trade credit facility retailer can order more quantity with minimal ordering cost and less investment capital. Also, retailer earns the interest on revenue accumulated by selling the items during the credit period.

While developing inventory models most of the authors assume the holding costs as constant. But it is observed that in some real inventory management, the holding cost of items in a rented warehouse (RW) increases over time or it is proportional to the demand of the rented warehouse (RW). For deteriorating items, the holding cost depends on the availing facility in the rented warehouse.

In the real business world, different costs associated with inventory models are imprecision in nature. That is to say, the holding cost, ordering cost, the interest rate and other associated costs are fluctuating over time. Thus, to deal with such inventory problems, fuzzy set theory has been an excellent tool.

Practically, various deteriorating inventory models have different deterioration patterns. For instance, in cloth items, electronic gadgets, construction materials, fruits, dry fruits, cold drinks, health drinks, medicines, different type of agricultural products, plastic products, metals have different deterioration patterns. Moreover, it has been observed that most of the daily needs have stipulated life span. For example, milk products, vegetables, cool drinks, packed foods, medicines etc. has short life span. Thus, retailer dealing with these type of items has always planned to sell them before they get expire. 
Considering the above mentioned facts, here we develop an inventory model having decreasing time dependant demand pattern with variable holding cost for Two-Storage facility under acceptable delay in payment. Moreover, the model is discussed in fuzzy environment by taking the parameters as trapezoidal fuzzy numbers. The objective of the work is to minimize the total cost of inventory by obtaining the optimal inventory time for both the warehouses in crisp and fuzzy environment, and thereafter to study the effect on the optimal solutions subject to the small changes in the associated parameters.

\section{Literature survey}

In the year 1976, Hartley [8] initially framed a Two-warehouse inventory problem. Whereas, the concept of trade credit financing in inventory was initiated by Haley and Higgins [7] in the year 1973. The two-warehouse inventory problem together with trade credit financing was developed by many researchers. Recently, Liang and Zhou [18], Liao et al. ( [19,20]), Bhunia et al. [4], Tiwari et al. [28], and Jaggi et al. [10] considered demand and holding cost as constant in their two warehouse inventory problems under permissible delay in payment. Further, the demand function is taken as stock and selling price dependent by Guchhait et al. [6], selling price dependent by Jaggi et al. [12,13] and Sing and Kumar [26], exponentially increasing by Kaliraman et al. [14], and Rajan and Uthayakumar [22] and ramp-type by Chakraborty et al. [5] with constant holding cost in their credit financing problem having two storage facility. But, Sett et al. [24], Yang [29], and Khurana [17] proposed two warehouse inventory models without trade credit financing by considering constant holding cost with different demands. Also, Khanna et al. [15,16] and Jaggi et al. [11] presented inventory models for single warehouse problems under allowable delay in payment with different demand patterns.

The different types of time dependent holding costs have been considered by Barik et al. [2,3], Alfares [1], Mishra and Mishra [21], and Routray et al. [23] for their single warehouse problem. But, Yu [30] considered decreasing holding cost of rented warehouse in his two warehouse inventory model having constant demand under trade credit offer.

In present scenario, most of the researchers using Fuzzy concept in their inventory model to deal the imprecise parameters. Recently, Singh et al. [27] developed a two warehouse inventory model for non deteriorating items with fuzzy demand and fuzzy holding cost without trade credit, Shabani et al. [25] considered fuzzy demand and constant holding cost for their two warehouse inventory problem under permissible delay in payment, Indrajitsingha et al. [9] developed two warehouse problem for items having selling price dependent demand and constant holding cost in fuzzy environment.

From the above mentioned literature, we found that most of the authors considered different types of demand for their single or two warehouse problems with or without trade credit financing. Among all the authors, Jaggi et al. [11] considered exponentially decreasing demand, but no author considered time varying decreasing demand. Again, most of the authors considered constant holding cost. Whereas, Barik et al. [2,3], Alfares [1], Mishra and 
Table 1. Major characteristics of recent inventory models discussed in the literature.

\begin{tabular}{|c|c|c|c|c|c|c|c|}
\hline Author(s) & Year & $\begin{array}{r}\text { Demand } \\
\text { Type }\end{array}$ & $\begin{array}{l}\text { Deterio } \\
\text {-ration }\end{array}$ & $\begin{array}{r}\text { Holding } \\
\text { Cost }\end{array}$ & $\begin{array}{r}\text { Stora } \\
\text {-ges }\end{array}$ & $\begin{array}{l}\text { Trade } \\
\text { credit }\end{array}$ & $\begin{array}{l}\text { Fuzzy } \\
\text { Model }\end{array}$ \\
\hline Singh et al. [27] & 2011 & Fuzzy & No & $\begin{array}{r}\text { Constant } \\
\& \text { fuzzy }\end{array}$ & Two & Yes & Yes \\
\hline Liang and Zhou [18] & 2011 & Constant & Yes & Constant & Two & Yes & No \\
\hline Liao et al. [20] & 2012 & Constant & Yes & Constant & Two & Yes & No \\
\hline Sett et al. [24] & 2012 & Quadratic & Yes & Constant & Two & No & No \\
\hline Yang H-L [29] & 2012 & Constant & Yes & Constant & Two & No & No \\
\hline Guchhait et al. [6] & 2013 & $\begin{array}{l}\text { Stock \& selling } \\
\text { price dependent }\end{array}$ & Yes & Constant & Two & Yes & Yes \\
\hline Liao et al. [19] & 2013 & Constant & Yes & Constant & Two & Yes & No \\
\hline Bhunia et al. [4] & 2014 & Constant & Yes & Constant & Two & Yes & No \\
\hline Jaggi et al. [12] & 2014 & $\begin{array}{r}\text { Selling price } \\
\text { dependent }\end{array}$ & Yes & Constant & Two & Yes & No \\
\hline Shabani et al. [25] & 2015 & Fuzzy & Yes & Constant & Two & Yes & Yes \\
\hline $\begin{array}{r}\text { Rajan and } \\
\text { Uthayakumar }[22]\end{array}$ & 2015 & $\begin{array}{r}\text { Exponential } \\
\text { Increasing }\end{array}$ & Yes & Constant & Two & Yes & No \\
\hline Khurana [17] & 2015 & $\begin{array}{r}\text { Time and } \\
\text { Stock dependent }\end{array}$ & Yes & Constant & Two & No & No \\
\hline Khanna et al. [15] & 2016 & Constant & Yes & Constant & One & Yes & No \\
\hline Tiwari et al. [28] & 2016 & Constant & Yes & Constant & Two & Yes & No \\
\hline Khanna et al. [16] & 2017 & $\begin{array}{r}\text { Selling price } \\
\text { dependent }\end{array}$ & Yes & Constant & One & Yes & No \\
\hline Jaggi et al. [13] & 2017 & $\begin{array}{r}\text { Selling price } \\
\text { dependent }\end{array}$ & Yes & Constant & Two & Yes & No \\
\hline Jaggi et al. [10] & 2017 & Constant & Yes & Constant & Two & Yes & No \\
\hline Kaliraman et al. [14] & 2017 & $\begin{array}{r}\text { Exponential } \\
\text { increasing }\end{array}$ & Yes & Constant & Two & Yes & No \\
\hline Jaggi et al. [11] & 2018 & $\begin{array}{r}\text { Exponentially } \\
\text { decreasing }\end{array}$ & Yes & Constant & One & Yes & No \\
\hline Chakraborty et al. [5] & 2018 & Ramp Type & Yes & Constant & Two & Yes & No \\
\hline $\begin{array}{r}\text { Singh and } \\
\text { Kumar [26] }\end{array}$ & 2018 & $\begin{array}{r}\text { Selling price } \\
\text { dependent }\end{array}$ & Yes & Constant & Two & Yes & No \\
\hline $\mathrm{Yu}[30]$ & 2019 & Constant & Yes & Decreasing & Two & Yes & No \\
\hline $\begin{array}{r}\text { Indrajitsingha } \\
\text { et al. }[9]\end{array}$ & 2019 & $\begin{array}{r}\text { Selling-Price } \\
\text { dependent }\end{array}$ & Yes & Constant & Two & No & Yes \\
\hline $\begin{array}{r}\text { Present } \\
\text { paper }\end{array}$ & & $\begin{array}{r}\text { Time varying } \\
\text { decreasing }\end{array}$ & No & $\begin{array}{r}\text { Variable } \\
\text { and Linear }\end{array}$ & Two & Yes & Yes \\
\hline
\end{tabular}


Mishra [21], Routray et al. [23] assumed variable holding cost for their single warehouse inventory problems and $\mathrm{Yu}$ [30] assumed decreasing holding cost for two-warehouse inventory problem. But, no one considered an increasing holding cost. Furthermore, some authors developed their inventory problems in fuzzy environment. Motivated essentially by the above mentioned results, here we investigate an optimal result for inventory items which follow decreasing demand and increasing rental cost with two warehouse facility under trade credit financing. Further, the model is treated in fuzzy environment to overcome the imprecision of associated costs. In Table 1, the precise comparison of the present model and the different models discussed in the literature are provided.

\section{Assumptions}

The following assumptions are carried out to develop the present model: (i) The homogeneous (or single) item is considered. (ii) The demand rate is decreasing over time. (iii) The associated items have a greater life span than that of cycle time $T$, and the number of items deteriorated during cycle is very negligible as compared to the stock in inventory. (iv) The instant Replenishment facility is available, that is, lead time is zero. (v) During the Inventory cycle, stock is available without any backlog, that is, shortages are not allowed. (vi) Twostorage facility is considered. Owned storage (OS) is the retailer's outlet and rented storage (RS) is away from the owned storage(OS). Moreover, the OS has limited storage capacity, and RS has unlimited capacity. (vii) The items in RS depletes first than that of the OS in order to reduce the cost of inventory. (viii)The holding cost of the OS is constant, but it varies with time for RS. Also, holding cost of RS is more than that of the OS (the holding cost of the RS includes transportation cost from RS to OS, loading and unloading costs). (ix) Supplier accepts the delay in payment by the retailer.

\section{$4 \quad$ Notations}

The following notations are used in the proposed model: $\mathbb{D}(t)$ : Demand rate $\left(\mathbb{D}(t)=\alpha t^{-\beta} \quad(\alpha>0,0<\beta<1)\right.$, where $\beta$ is the demand parameter $)$. $\mathcal{A}$ : Ordering cost per cycle. $\mathcal{P C}$ : Purchasing cost per unit item. $\mathcal{S P}$ : Selling price per unit item. $\mathrm{H}(\mathrm{t})$ : Holding cost per unit item $\left(\mathcal{H}_{o}\right.$, constant for OS and $\mathcal{H}_{r} * t+b$, variable for $\left.\mathrm{RS}, b \geq \mathcal{H}_{o}\right)$. W: The maximum number of items can be ordered at the beginning of the cycle (at most, W1 items are kept in the OS, remaining in $\mathrm{RS}) . \mathcal{Q}_{r}[t]$ : The inventory level at any time $t$ in $\mathrm{RS}$. $\mathcal{Q}_{o}[t]$ : The inventory level at any time $t$ in OS. $\mathcal{M}$ : Acceptable delay period offered by the supplier to the retailer. $\vartheta$ : The rate of interest earned by the retailer on the amount obtained by selling the items during the acceptable delay of the payment period. $\theta$ : The rate of interest payable by the retailer on the amount of stock left in the inventory after an acceptable delay of the payment period. $\tau$ : Time at which inventory in RS falls to zero. T: Time at which inventory in OS falls to zero, that is, both the storages are empty. $\mathbb{Z}(\tau)$ : Total cost of 
inventory cycle. $\mathbb{Z} 1(\tau)$ : Total cost of inventory for $\mathcal{M} \leq \tau \leq T$. $\mathbb{Z} 2(\tau)$ : Total cost of inventory for $\tau<\mathcal{M} \leq T$. $\mathbb{Z} 3(\tau)$ : Total cost of inventory for $\mathcal{M}>T$.

Now, $\tilde{\vartheta}, \tilde{\theta}, \tilde{\mathcal{H}}_{o}, \tilde{\mathcal{H}}_{r}, \tilde{\mathbb{Z}}(\tau), \tilde{\mathbb{Z}} 1(\tau), \tilde{\mathbb{Z}} 2(\tau)$, and $\tilde{\mathbb{Z}} 3(\tau)$ are used for $\vartheta, \theta, \mathcal{H}_{o}$, $\mathcal{H}_{r}, \mathbb{Z}(\tau), \mathbb{Z} 1(\tau), \mathbb{Z} 2(\tau)$ and $\mathbb{Z} 3(\tau)$ respectively in fuzzy environment. Also, $\operatorname{GMZ}(\tau), \operatorname{GM} \mathbb{Z} 1(\tau), \operatorname{GM} \mathbb{Z} 2(\tau)$, and $\operatorname{GMZ} 3(\tau)$ are used to represent defuzzified total costs.

\section{$5 \quad$ Mathematical model}

In the present model, initially $\mathrm{W}$ amount of items ordered to the supplier. As the lead time is zero, the $\mathrm{W}$ items (W1 items are kept in OS and remaining (W-W1) in RS) are delivered instantly to the retailer. To reduce the cost of inventory the retailer sells the items in RS first, then OS. So during the interval $[0, \tau]$, the inventory in RS is gradually reduces due to demand of the items, and is vanishing at time $t=\tau$. After RS is empty, the inventory in OS gradually decreases during the interval $[\tau, T]$, and is vanishing at time $t=T$. That means, both the storages are empty at time $T$. Figure 1 shows the inventory level at any time $t$. During the time $t \in[0, \tau]$, the inventory in RS is gradually

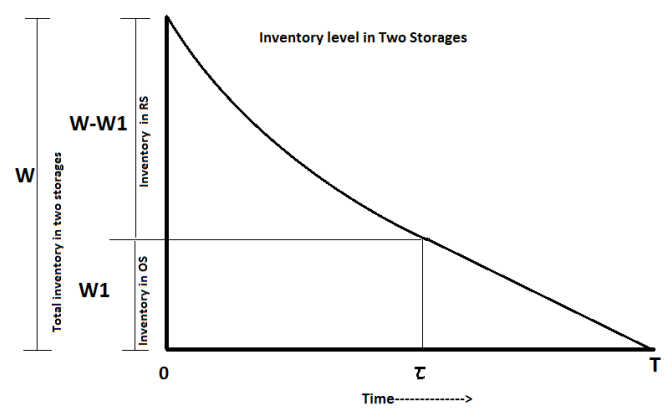

Figure 1. Inventory level at any time $t$ in both the Storages.

decreases, and reaches at 0 at $t=\tau$. The inventory level in RS is governed by the differential equation

$$
\frac{d \mathcal{Q}_{r}[t]}{d t}=-\alpha t^{-\beta} \quad(0<t \leq \tau)
$$

under the boundary condition $\mathcal{Q}_{r}[\tau]=0$. Solving this equation, we have

$$
\mathcal{Q}_{o}[t]=-\alpha t^{-\beta} T^{-\beta}\left(T t^{\beta}-t T^{\beta}\right) /(\beta-1) .
$$

Next, during the time $t \in[0, \tau]$, the inventory in OS becomes constant, and for $t \in[\tau, T]$ it gradually decreases and vanishes at $t=T$. The inventory level in OS is governed by the differential equation

$$
\frac{d \mathcal{Q}_{o}[t]}{d t}=-\alpha t^{-\beta} \quad(\tau \leq t \leq T)
$$


under the boundary condition $\mathcal{Q}_{o}[T]=0$. Solving this equation, we have

$$
\mathcal{Q}_{o}[t]=-\alpha t^{-\beta} T^{-\beta}\left(T t^{\beta}-t T^{\beta}\right) /(\beta-1) .
$$

At time $t=\tau$ the amount of inventory in OS is W1. Thus, we have

$$
-\alpha \tau^{-\beta} T^{-\beta}\left(T \tau^{\beta}-\tau T^{\beta}\right) /(\beta-1)=\mathrm{W} 1 .
$$

Solving this equation for $T$, we have

$$
T=\left(-(1-\beta)\left(-\frac{\alpha \tau^{1-\beta}}{1-\beta}-\mathrm{W} 1\right) / \alpha\right)^{1 /(1-\beta)} .
$$

Based on the assumptions in the model, the total relevant cost $\mathbb{Z}(\tau)$ of inventory includes the ordering cost, holding cost, interest earned on sales amount during the trade credit period and interest payable on stock in the inventory after allowable delay time. Now the costs are calculated as follows.

(i) Ordering Cost, $\mathrm{OC}=\mathcal{A} / T$.

(ii) Total Stock Holding Cost,

$$
\begin{aligned}
\mathrm{SHC} & =\frac{\mathcal{H}_{o}}{T} \tau \mathrm{W} 1+\frac{\mathcal{H}_{o}}{T} \int_{\tau}^{T} \mathcal{Q}_{o}(t) d t+\int_{0}^{\tau}\left(\frac{\mathcal{H}_{r} t+b}{T}\right) \mathcal{Q}_{r}[t] d t \\
= & \frac{\mathcal{H}_{o}}{T} \tau \mathrm{W} 1+\frac{\mathcal{H}_{o}}{T}\left(-\frac{\alpha T^{2-\beta}}{\beta-1}+\frac{\alpha \tau T^{1-\beta}}{\beta-1}+\frac{\alpha}{\beta-1}\left(-\frac{T^{2-\beta}}{\beta-2}+\frac{\tau^{2-\beta}}{\beta-2}\right)\right) \\
& +\frac{\alpha \tau^{2-\beta}}{2(T-T \beta)}\left(2 b\left(1+\frac{1}{\beta-2}\right)+\frac{(\beta-1) \tau \mathcal{H}_{r}}{\beta-3}\right) .
\end{aligned}
$$

(iii) Interest Payable by Retailer; there arise three cases:

Case $1 \quad(\mathcal{M} \leq \tau \leq T)$ :

$$
\begin{aligned}
\mathrm{IP} 1= & \frac{(\mathcal{P C} \theta)}{T}\left(\int_{\mathcal{M}}^{\tau} \mathcal{Q}_{r}[t] d t+\int_{0}^{T} \mathcal{Q}_{o}[t] d t\right) \\
= & \frac{(\mathcal{P C} \theta)}{T}\left(\frac{\alpha}{1-\beta}\left(\frac{T^{2-\beta}}{\beta-2}+T^{2-\beta}\right)+\frac{\alpha \mathcal{M} \tau^{1-\beta}}{\beta-1}-\frac{\alpha \tau^{2-\beta}}{\beta-1}\right. \\
& \left.+\frac{\alpha}{\beta-1}\left(\frac{\mathcal{M}^{2-\beta}}{\beta-2}-\frac{\tau^{2-\beta}}{\beta-2}\right)\right) .
\end{aligned}
$$

Case $2(\tau<\mathcal{M} \leq T)$ :

$$
\begin{aligned}
\mathrm{IP} 2 & =\frac{\mathcal{P C} \theta}{T} \int_{\mathcal{M}}^{T} \mathcal{Q}_{o}[t] d t \\
& =\frac{\mathcal{P C} \theta}{T}\left(-\frac{\alpha T^{2-\beta}}{\beta-1}+\frac{\alpha \mathcal{M} T^{1-\beta}}{\beta-1}+\frac{\alpha}{\beta-1}\left(\frac{\mathcal{M}^{2-\beta}}{\beta-2}-\frac{T^{2-\beta}}{\beta-2}\right)\right) .
\end{aligned}
$$

Case $3(\mathcal{M}>T):$ IP3 $=0$. 
(iv) Interest earned by Retailer; there arise two cases:

Case $1 \quad(\mathcal{M} \leq T)$ :

$$
\mathrm{IE} 1=\frac{(\mathcal{S P} \vartheta)}{T} \int_{0}^{\mathcal{M}} t \mathbb{D}(t) d t=-\frac{(\mathcal{S P} \vartheta)}{T}\left(\frac{\alpha \mathcal{M}^{2-\beta}}{\beta-2}\right) .
$$

Case $2(\mathcal{M}>T)$ :

$$
\begin{aligned}
\mathrm{IE} 2 & =\frac{\mathcal{S P} \vartheta}{T}\left(\int_{0}^{T} t \mathbb{D}(t) d t+(\mathcal{M}-T) \int_{0}^{T} \mathbb{D}(t) d t\right) \\
& =\frac{\mathcal{S P} \vartheta}{T}\left(-\frac{\alpha T^{2-\beta}}{\beta-2}-\frac{\alpha T^{1-\beta}(\mathcal{M}-T)}{\beta-1}\right) .
\end{aligned}
$$

Thus the total relevant cost of the inventory per year is given by, $\mathbb{Z}(\tau)=$ Ordering Cost + Total Stock Holding Cost + Interest payable - Interest Earned. Moreover, according to the acceptable delay period given by the supplier, the total cost function $\mathbb{Z}(\tau)$ is given by,

$$
\mathbb{Z}(\tau)= \begin{cases}\mathbb{Z} 1(\tau), & \mathcal{M} \leq \tau<T, \\ \mathbb{Z} 2(\tau), & \tau<\mathcal{M} \leq T, \\ \mathbb{Z} 3(\tau), & \tau>T .\end{cases}
$$

We have,

$$
\begin{aligned}
\mathbb{Z} 1(\tau)= & O C+S H C+I P 1-I E 1 \\
= & \frac{\mathcal{A}}{T}+\frac{\mathcal{H}_{o}}{T} \tau \mathrm{W} 1+\frac{\mathcal{H}_{o}}{T}\left(-\frac{\alpha T^{2-\beta}}{\beta-1}+\frac{\alpha \tau T^{1-\beta}}{\beta-1}+\frac{\alpha}{\beta-1}\left(-\frac{T^{2-\beta}}{\beta-2}\right.\right. \\
& \left.\left.+\frac{\tau^{2-\beta}}{\beta-2}\right)\right)+\frac{\alpha \tau^{2-\beta}}{2(T-T \beta)}\left(2 b\left(1+\frac{1}{\beta-2}\right)+\frac{(\beta-1) \tau \mathcal{H}_{r}}{\beta-3}\right) \\
& +\frac{(\mathcal{P C} \theta)}{T}\left(\frac{\alpha}{1-\beta}\left(\frac{T^{2-\beta}}{\beta-2}+T^{2-\beta}\right)+\frac{\alpha \mathcal{M} \tau^{1-\beta}}{\beta-1}-\frac{\alpha \tau^{2-\beta}}{\beta-1}\right. \\
& \left.+\frac{\alpha}{\beta-1}\left(\frac{\mathcal{M}^{2-\beta}}{\beta-2}-\frac{\tau^{2-\beta}}{\beta-2}\right)\right)-\left(-\frac{(\mathcal{S P} \vartheta)}{T}\left(\frac{\alpha \mathcal{M}^{2-\beta}}{\beta-2}\right)\right) . \\
\mathbb{Z} 2(\tau)= & O C+S H C+I P 2-I E 1 \\
& \frac{\mathcal{A}}{T}+\frac{\mathcal{H}}{T} \tau \mathrm{W} 1+\frac{\mathcal{H}_{o}}{T}\left(-\frac{\alpha T^{2-\beta}}{\beta-1}+\frac{\alpha \tau T^{1-\beta}}{\beta-1}+\frac{\alpha}{\beta-1}\left(-\frac{T^{2-\beta}}{\beta-2}\right.\right. \\
& \left.\left.+\frac{\tau^{2-\beta}}{\beta-2}\right)\right)+\frac{\alpha \tau^{2-\beta}}{2(T-T \beta)}\left(2 b\left(1+\frac{1}{\beta-2}\right)+\frac{(\beta-1) \tau \mathcal{H}_{r}}{\beta-3}\right) \\
& +\frac{\mathcal{P C} \theta}{T}\left(-\frac{\alpha T^{2-\beta}}{\beta-1}+\frac{\alpha \mathcal{M} T^{1-\beta}}{\beta-1}+\frac{\alpha}{\beta-1}\left(\frac{\mathcal{M}^{2-\beta}}{\beta-2}-\frac{T^{2-\beta}}{\beta-2}\right)\right) \\
& -\left(-\frac{\mathcal{S} \mathcal{P} \vartheta)}{T}\left(\frac{\alpha \mathcal{M}}{\beta-\beta}\right)\right) . \\
& \left.\left(\frac{\beta-2}{\beta-2}\right)\right)
\end{aligned}
$$




$$
\begin{aligned}
\mathbb{Z} 3(\tau)= & O C+S H C+I P 3-I E 2 \\
= & \frac{\mathcal{A}}{T}+\frac{\mathcal{H}_{o}}{T} \tau \mathrm{W} 1+\frac{\mathcal{H}_{o}}{T}\left(-\frac{\alpha T^{2-\beta}}{\beta-1}+\frac{\alpha \tau T^{1-\beta}}{\beta-1}+\frac{\alpha}{\beta-1}\left(-\frac{T^{2-\beta}}{\beta-2}\right.\right. \\
& \left.\left.+\frac{\tau^{2-\beta}}{\beta-2}\right)\right)+\frac{\alpha \tau^{2-\beta}}{2(T-T \beta)}\left(2 b\left(1+\frac{1}{\beta-2}\right)+\frac{(\beta-1) \tau \mathcal{H}_{r}}{\beta-3}\right)+0 \\
& -\left(\frac{\mathcal{S P} \vartheta}{T}\left(-\frac{\alpha T^{2-\beta}}{\beta-2}-\frac{\alpha T^{1-\beta}(\mathcal{M}-T)}{\beta-1}\right)\right) .
\end{aligned}
$$

\section{Solution procedure}

The aim of the present model is to find the optimal value of $\tau$ such that $\mathbb{Z}(\tau)$ is minimum. The working rule is as follows:

(i) Use the expression for $T$ from equation (5.1) in $\mathbb{Z} 1(\tau), \mathbb{Z} 2(\tau)$ and $\mathbb{Z} 3(\tau)$.

(ii) Solve the equation $\frac{d \mathbb{Z} 1}{d \tau}=0$ for $\tau$. This $\tau$ can be the optimal value for $\mathbb{Z} 1(\tau)$, if $\frac{d^{2} \mathbb{Z} 1}{d \tau^{2}}>0$. If so, find $T$ from equation (5.1) and set $\tau_{1}^{*}=$ $\tau \& T_{1}^{*}=T$.

(iii) Solve the equation $\frac{d \mathbb{Z} 2}{d \tau}=0$ for $\tau$. This $\tau$ can be the optimal value for $\mathbb{Z} 2(\tau)$, if $\frac{d^{2} \mathbb{Z} 2}{d \tau^{2}}>0$. If so, find $T$ from equation (5.1) and set $\tau_{2}^{*}=$ $\tau \& T_{2}^{*}=T$.

(iv) Solve the equation $\frac{d \mathbb{Z} 3}{d \tau}=0$ for $\tau$. This $\tau$ can be the optimal value for $\mathbb{Z} 3(\tau)$, if $\frac{d^{2} \mathbb{Z} 3}{d \tau^{2}}>0$. If so, find $T$ from equation (5.1) and set $\tau_{3}^{*}=$ $\tau \& T_{3}^{*}=T$.

\section{Algorithm to find the optimal solution}

Step-1. If $\mathcal{M} \leq \tau_{1}^{*} \leq T_{1}^{*}$, then set $\tau^{*}=\tau_{1}^{*}, T^{*}=T_{1}^{*}$. Find $\mathbb{Z}^{*}(\tau)=\mathbb{Z} 1\left(\tau^{*}\right)$; otherwise go to step-2.

Step-2. If $\tau_{2}^{*}<\mathcal{M} \leq T_{2}^{*}$, then set $\tau^{*}=\tau_{2}^{*}, T^{*}=T_{2}^{*}$. Find $\mathbb{Z}^{*}(\tau)=\mathbb{Z} 2\left(\tau^{*}\right)$; otherwise go to step-3.

Step-3. If $\tau_{3}^{*}<T_{3}^{*}<\mathcal{M}$, then set $\tau^{*}=\tau_{3}^{*}, T^{*}=T_{3}^{*}$. Find $\mathbb{Z}^{*}(\tau)=\mathbb{Z} 3\left(\tau^{*}\right)$; otherwise go to step-4.

Step-4. If all the above cases fail, then $\mathbb{Z}^{*}(\tau)=\min \left\{\mathbb{Z} 1\left(\tau_{1}^{*}\right), \mathbb{Z} 2\left(\tau_{2}^{*}\right), \mathbb{Z} 3\left(\tau_{3}^{*}\right)\right\}$ and $\tau^{*}=\operatorname{argmin}\left\{\mathbb{Z} 1\left(\tau_{1}^{*}\right), \mathbb{Z} 2\left(\tau_{2}^{*}\right), \mathbb{Z} 3\left(\tau_{3}^{*}\right)\right\} . T^{*}$ can be obtained from the corresponding $\tau^{*}$.

Then the optimal solutions are $\tau^{*}, T^{*} \& \mathbb{Z}^{*}(\tau)$.

\section{$7 \quad$ Fuzzy model}

In the real world business, the different costs associated with any inventory model vary time to time. In this model, the holding cost of items $\mathcal{H}_{r}$ (for 
$\mathrm{RS}$ ) and $\mathcal{H}_{o}$ (for OS), the interest payable $\theta$ and interest earned $\vartheta$ rates are not constant. Thus, we consider them as Fuzzy numbers $\tilde{\mathcal{H}}_{r}, \tilde{\mathcal{H}}_{o}, \tilde{\theta}$ and $\tilde{\vartheta}$ respectively. Taking all these parameters in to consideration, the total cost function under fuzzy environment is given by

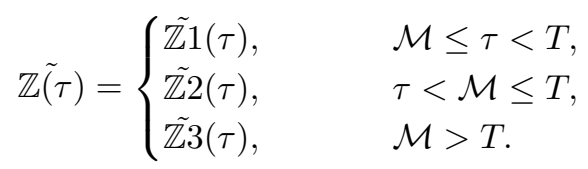

Here, we have

$$
\begin{aligned}
& \tilde{\mathbb{Z}} 1(\tau)=\frac{\mathcal{A}}{T}+\frac{\tilde{\mathcal{H}}_{o}}{T} \tau \mathrm{W} 1+\frac{\tilde{\mathcal{H}}_{o}}{T}\left(-\frac{\alpha T^{2-\beta}}{\beta-1}+\frac{\alpha \tau T^{1-\beta}}{\beta-1}+\frac{\alpha}{\beta-1}\left(-\frac{T^{2-\beta}}{\beta-2}\right.\right. \\
& \left.\left.+\frac{\tau^{2-\beta}}{\beta-2}\right)\right)+\frac{\alpha \tau^{2-\beta}}{2(T-T \beta)}\left(2 b\left(1+\frac{1}{\beta-2}\right)+\frac{(\beta-1) \tau \tilde{\mathcal{H}}_{r}}{\beta-3}\right) \\
& +\frac{(\mathcal{P C} \tilde{\theta})}{T}\left(\frac{\alpha}{1-\beta}\left(\frac{T^{2-\beta}}{\beta-2}+T^{2-\beta}\right)+\frac{\alpha \mathcal{M} \tau^{1-\beta}}{\beta-1}-\frac{\alpha \tau^{2-\beta}}{\beta-1}\right. \\
& \left.+\frac{\alpha}{\beta-1}\left(\frac{\mathcal{M}^{2-\beta}}{\beta-2}-\frac{\tau^{2-\beta}}{\beta-2}\right)\right)-\left(-\frac{(\mathcal{S P} \tilde{\vartheta})}{T}\left(\frac{\alpha \mathcal{M}^{2-\beta}}{\beta-2}\right)\right) \text {. } \\
& \tilde{\mathbb{Z}} 2(\tau)=\frac{\mathcal{A}}{T}+\frac{\tilde{\mathcal{H}}_{o}}{T} \tau \mathrm{W} 1+\frac{\tilde{\mathcal{H}}_{o}}{T}\left(-\frac{\alpha T^{2-\beta}}{\beta-1}+\frac{\alpha \tau T^{1-\beta}}{\beta-1}+\frac{\alpha}{\beta-1}\left(-\frac{T^{2-\beta}}{\beta-2}\right.\right. \\
& \left.\left.+\frac{\tau^{2-\beta}}{\beta-2}\right)\right)+\frac{\alpha \tau^{2-\beta}}{2(T-T \beta)}\left(2 b\left(1+\frac{1}{\beta-2}\right)+\frac{(\beta-1) \tau \tilde{\mathcal{H}}_{r}}{\beta-3}\right) \\
& +\frac{\mathcal{P C} \tilde{\theta}}{T}\left(-\frac{\alpha T^{2-\beta}}{\beta-1}+\frac{\alpha \mathcal{M} T^{1-\beta}}{\beta-1}+\frac{\alpha}{\beta-1}\left(\frac{\mathcal{M}^{2-\beta}}{\beta-2}-\frac{T^{2-\beta}}{\beta-2}\right)\right) \\
& -\left(-\frac{(\mathcal{S P} \tilde{\vartheta})}{T}\left(\frac{\alpha \mathcal{M}^{2-\beta}}{\beta-2}\right)\right) \text {. } \\
& \tilde{\mathbb{Z}} 3(\tau)=\frac{\mathcal{A}}{T}+\frac{\tilde{\mathcal{H}}_{o}}{T} \tau \mathrm{W} 1+\frac{\tilde{\mathcal{H}}_{o}}{T}\left(-\frac{\alpha T^{2-\beta}}{\beta-1}+\frac{\alpha \tau T^{1-\beta}}{\beta-1}+\frac{\alpha}{\beta-1}\left(-\frac{T^{2-\beta}}{\beta-2}\right.\right. \\
& \left.\left.+\frac{\tau^{2-\beta}}{\beta-2}\right)\right)+\frac{\alpha \tau^{2-\beta}}{2(T-T \beta)}\left(2 b\left(1+\frac{1}{\beta-2}\right)+\frac{(\beta-1) \tau \tilde{\mathcal{H}}_{r}}{\beta-3}\right)+0 \\
& -\left(\frac{\mathcal{S P} \tilde{\vartheta}}{T}\left(-\frac{\alpha T^{2-\beta}}{\beta-2}-\frac{\alpha T^{1-\beta}(\mathcal{M}-T)}{\beta-1}\right)\right) \text {. }
\end{aligned}
$$

In particular, let $\tilde{\theta}=(\theta 1, \theta 2, \theta 3, \theta 4), \tilde{\mathcal{H}}_{r}=(H R 1, H R 2, H R 3, H R 4), \tilde{\mathcal{H}}_{o}=$ (HO1, HO2, HO3, HO4), $\tilde{\vartheta}=(\vartheta 1, \vartheta 2, \vartheta 3, \vartheta 4)$ be the trapezoidal fuzzy numbers. Thus, the total cost functions (fuzzy) after defuzzification by Graded Mean Integration Representation (GMIR) Method, we have

$$
G M \mathbb{Z} i=\frac{1}{6}\left[G M \mathbb{Z}_{i 1}+2 G M \mathbb{Z}_{i 2}+2 G M \mathbb{Z}_{i 3}+G M \mathbb{Z}_{i 4}\right] \text { for } i=1,2,3
$$


Now

$$
\begin{aligned}
& G M \mathbb{Z} 1=\frac{1}{6}\left\{\left[\frac{\mathcal{A}}{T}+\frac{H O 1}{T} \tau \mathrm{W} 1+\frac{H O 1}{T}\left(-\frac{\alpha T^{2-\beta}}{\beta-1}+\frac{\alpha \tau T^{1-\beta}}{\beta-1}+\frac{\alpha}{\beta-1}\right.\right.\right. \\
& \left.\left(-\frac{T^{2-\beta}}{\beta-2}+\frac{\tau^{2-\beta}}{\beta-2}\right)\right)+\frac{\alpha \tau^{2-\beta}}{2(T-T \beta)}\left(2 b\left(1+\frac{1}{\beta-2}\right)+\frac{(\beta-1) \tau H R 1}{\beta-3}\right) \\
& +\frac{(\mathcal{P C} \theta 1)}{T}\left(\frac{\alpha}{1-\beta}\left(\frac{T^{2-\beta}}{\beta-2}+T^{2-\beta}\right)+\frac{\alpha \mathcal{M} \tau^{1-\beta}}{\beta-1}-\frac{\alpha \tau^{2-\beta}}{\beta-1}+\frac{\alpha}{\beta-1}\left(\frac{\mathcal{M}^{2-\beta}}{\beta-2}-\frac{\tau^{2-\beta}}{\beta-2}\right)\right) \\
& \left.-\left(-\frac{(\mathcal{S P} \vartheta 1)}{T}\left(\frac{\alpha \mathcal{M}^{2-\beta}}{\beta-2}\right)\right)\right]+2\left[\frac{\mathcal{A}}{T}+\frac{H O 2}{T} \tau \mathrm{W} 1+\frac{H O 2}{T}\left(-\frac{\alpha T^{2-\beta}}{\beta-1}\right.\right. \\
& \left.+\frac{\alpha \tau T^{1-\beta}}{\beta-1}+\frac{\alpha}{\beta-1}\left(-\frac{T^{2-\beta}}{\beta-2}+\frac{\tau^{2-\beta}}{\beta-2}\right)\right)+\frac{\alpha \tau^{2-\beta}}{2(T-T \beta)}\left(2 b\left(1+\frac{1}{\beta-2}\right)\right. \\
& \left.+\frac{(\beta-1) \tau H R 2}{\beta-3}\right)+\frac{(\mathcal{P C} \theta 2)}{T}\left(\frac{\alpha}{1-\beta}\left(\frac{T^{2-\beta}}{\beta-2}+T^{2-\beta}\right)+\frac{\alpha \mathcal{M} \tau^{1-\beta}}{\beta-1}-\frac{\alpha \tau^{2-\beta}}{\beta-1}\right. \\
& \left.\left.+\frac{\alpha}{\beta-1}\left(\frac{\mathcal{M}^{2-\beta}}{\beta-2}-\frac{\tau^{2-\beta}}{\beta-2}\right)\right)-\left(-\frac{(\mathcal{S P} \vartheta 2)}{T}\left(\frac{\alpha \mathcal{M}^{2-\beta}}{\beta-2}\right)\right)\right]+2\left[\frac{\mathcal{A}}{T}+\frac{H O 3}{T} \tau \mathrm{W} 1\right. \\
& +\frac{H O 3}{T}\left(-\frac{\alpha T^{2-\beta}}{\beta-1}+\frac{\alpha \tau T^{1-\beta}}{\beta-1}+\frac{\alpha}{\beta-1}\left(-\frac{T^{2-\beta}}{\beta-2}+\frac{\tau^{2-\beta}}{\beta-2}\right)\right) \\
& +\frac{\alpha \tau^{2-\beta}}{2(T-T \beta)}\left(2 b\left(1+\frac{1}{\beta-2}\right)+\frac{(\beta-1) \tau H R 3}{\beta-3}\right)+\frac{(\mathcal{P C} \theta 3)}{T} \\
& \times\left(\frac{\alpha}{1-\beta}\left(\frac{T^{2-\beta}}{\beta-2}+T^{2-\beta}\right)+\frac{\alpha \mathcal{M} \tau^{1-\beta}}{\beta-1}-\frac{\alpha \tau^{2-\beta}}{\beta-1}+\frac{\alpha}{\beta-1}\left(\frac{\mathcal{M}^{2-\beta}}{\beta-2}-\frac{\tau^{2-\beta}}{\beta-2}\right)\right) \\
& \left.-\left(-\frac{(\mathcal{S P} \vartheta 3)}{T}\left(\frac{\alpha \mathcal{M}^{2-\beta}}{\beta-2}\right)\right)\right]+\left[\frac{\mathcal{A}}{T}+\frac{H O 4}{T} \tau \mathrm{W} 1+\frac{H O 4}{T}\left(-\frac{\alpha T^{2-\beta}}{\beta-1}+\frac{\alpha \tau T^{1-\beta}}{\beta-1}\right.\right. \\
& \left.+\frac{\alpha}{\beta-1}\left(-\frac{T^{2-\beta}}{\beta-2}+\frac{\tau^{2-\beta}}{\beta-2}\right)\right)+\frac{\alpha \tau^{2-\beta}}{2(T-T \beta)}\left(2 b\left(1+\frac{1}{\beta-2}\right)\right. \\
& \left.+\frac{(\beta-1) \tau H R 4}{\beta-3}\right)+\frac{(\mathcal{P C} \theta 4)}{T}\left(\frac{\alpha}{1-\beta}\left(\frac{T^{2-\beta}}{\beta-2}+T^{2-\beta}\right)+\frac{\alpha \mathcal{M} \tau^{1-\beta}}{\beta-1}-\frac{\alpha \tau^{2-\beta}}{\beta-1}\right. \\
& \left.\left.\left.+\frac{\alpha}{\beta-1}\left(\frac{\mathcal{M}^{2-\beta}}{\beta-2}-\frac{\tau^{2-\beta}}{\beta-2}\right)\right)-\left(-\frac{(\mathcal{S P} \vartheta)}{T}\left(\frac{\alpha \mathcal{M}^{2-\beta}}{\beta-2}\right)\right)\right]\right\} \text {, }
\end{aligned}
$$$$
G M \mathbb{Z} 2=\frac{1}{6}\left\{\left[\frac{\mathcal{A}}{T}+\frac{H O 1}{T} \tau \mathrm{W} 1+\frac{H O 1}{T}\left(-\frac{\alpha T^{2-\beta}}{\beta-1}+\frac{\alpha \tau T^{1-\beta}}{\beta-1}+\frac{\alpha}{\beta-1}\right.\right.\right.
$$$$
\left.\times\left(-\frac{T^{2-\beta}}{\beta-2}+\frac{\tau^{2-\beta}}{\beta-2}\right)\right)+\frac{\alpha \tau^{2-\beta}}{2(T-T \beta)}\left(2 b\left(1+\frac{1}{\beta-2}\right)+\frac{(\beta-1) \tau H R 1}{\beta-3}\right)
$$$$
+\frac{\mathcal{P C} \theta 1}{T}\left(-\frac{\alpha T^{2-\beta}}{\beta-1}+\frac{\alpha \mathcal{M} T^{1-\beta}}{\beta-1}+\frac{\alpha}{\beta-1}\left(\frac{\mathcal{M}^{2-\beta}}{\beta-2}-\frac{T^{2-\beta}}{\beta-2}\right)\right)
$$$$
\left.-\left(-\frac{(\mathcal{S P} \vartheta 1)}{T}\left(\frac{\alpha \mathcal{M}^{2-\beta}}{\beta-2}\right)\right)\right]+2\left[\frac{\mathcal{A}}{T}+\frac{H O 2}{T} \tau \mathrm{W} 1+\frac{H O 2}{T}\left(-\frac{\alpha T^{2-\beta}}{\beta-1}\right.\right.
$$$$
\left.+\frac{\alpha \tau T^{1-\beta}}{\beta-1}+\frac{\alpha}{\beta-1}\left(-\frac{T^{2-\beta}}{\beta-2}+\frac{\tau^{2-\beta}}{\beta-2}\right)\right)+\frac{\alpha \tau^{2-\beta}}{2(T-T \beta)}\left(2 b\left(1+\frac{1}{\beta-2}\right)\right.
$$ 


$$
\begin{aligned}
& \left.+\frac{(\beta-1) \tau H R 2}{\beta-3}\right)+\frac{\mathcal{P C} \theta 2}{T}\left(-\frac{\alpha T^{2-\beta}}{\beta-1}+\frac{\alpha \mathcal{M} T^{1-\beta}}{\beta-1}+\frac{\alpha}{\beta-1}\left(\frac{\mathcal{M}^{2-\beta}}{\beta-2}-\frac{T^{2-\beta}}{\beta-2}\right)\right) \\
& \left.-\left(-\frac{(\mathcal{S P} \vartheta 2)}{T}\left(\frac{\alpha \mathcal{M}^{2-\beta}}{\beta-2}\right)\right)\right]+2\left[\frac{\mathcal{A}}{T}+\frac{H O 3}{T} \tau \mathrm{W} 1+\frac{H O 3}{T}\left(-\frac{\alpha T^{2-\beta}}{\beta-1}\right.\right. \\
& \left.+\frac{\alpha \tau T^{1-\beta}}{\beta-1}+\frac{\alpha}{\beta-1}\left(-\frac{T^{2-\beta}}{\beta-2}+\frac{\tau^{2-\beta}}{\beta-2}\right)\right)+\frac{\alpha \tau^{2-\beta}}{2(T-T \beta)}\left(2 b\left(1+\frac{1}{\beta-2}\right)\right. \\
& \left.+\frac{(\beta-1) \tau H R 3}{\beta-3}\right)+\frac{\mathcal{P C} \theta 3}{T}\left(-\frac{\alpha T^{2-\beta}}{\beta-1}+\frac{\alpha \mathcal{M} T^{1-\beta}}{\beta-1}+\frac{\alpha}{\beta-1}\left(\frac{\mathcal{M}^{2-\beta}}{\beta-2}-\frac{T^{2-\beta}}{\beta-2}\right)\right) \\
& \left.-\left(-\frac{(\mathcal{S P} \vartheta 3)}{T}\left(\frac{\alpha \mathcal{M}^{2-\beta}}{\beta-2}\right)\right)\right]+\left[\frac{\mathcal{A}}{T}+\frac{H O 4}{T} \tau \mathrm{W} 1+\frac{H O 4}{T}\left(-\frac{\alpha T^{2-\beta}}{\beta-1}\right.\right. \\
& \left.+\frac{\alpha \tau T^{1-\beta}}{\beta-1}+\frac{\alpha}{\beta-1}\left(-\frac{T^{2-\beta}}{\beta-2}+\frac{\tau^{2-\beta}}{\beta-2}\right)\right)+\frac{\alpha \tau^{2-\beta}}{2(T-T \beta)}\left(2 b\left(1+\frac{1}{\beta-2}\right)\right. \\
& \left.+\frac{(\beta-1) \tau H R 4}{\beta-3}\right)+\frac{\mathcal{P C} \theta 4}{T}\left(-\frac{\alpha T^{2-\beta}}{\beta-1}+\frac{\alpha \mathcal{M} T^{1-\beta}}{\beta-1}\right. \\
& \left.\left.\left.+\frac{\alpha}{\beta-1}\left(\frac{\mathcal{M}^{2-\beta}}{\beta-2}-\frac{T^{2-\beta}}{\beta-2}\right)\right)-\left(-\frac{(\mathcal{S P} \vartheta 4)}{T}\left(\frac{\alpha \mathcal{M} \mathcal{M}^{2-\beta}}{\beta-2}\right)\right)\right]\right\},
\end{aligned}
$$

$G M \mathbb{Z} 3=\frac{1}{6}\left\{\left[\frac{\mathcal{A}}{T}+\frac{H O 1}{T} \tau \mathrm{W} 1+\frac{H O 1}{T}\left(-\frac{\alpha T^{2-\beta}}{\beta-1}+\frac{\alpha \tau T^{1-\beta}}{\beta-1}+\frac{\alpha}{\beta-1}\right.\right.\right.$

$\left.\times\left(-\frac{T^{2-\beta}}{\beta-2}+\frac{\tau^{2-\beta}}{\beta-2}\right)\right)+\frac{\alpha \tau^{2-\beta}}{2(T-T \beta)}\left(2 b\left(1+\frac{1}{\beta-2}\right)+\frac{(\beta-1) \tau H R 1}{\beta-3}\right)$

$\left.-\left(\frac{\mathcal{S P} \vartheta 1}{T}\left(-\frac{\alpha T^{2-\beta}}{\beta-2}-\frac{\alpha T^{1-\beta}(\mathcal{M}-T)}{\beta-1}\right)\right)\right]+2\left[\frac{\mathcal{A}}{T}+\frac{H O 2}{T} \tau \mathrm{W} 1+\frac{H O 2}{T}\right.$

$\times\left(-\frac{\alpha T^{2-\beta}}{\beta-1}+\frac{\alpha \tau T^{1-\beta}}{\beta-1}+\frac{\alpha}{\beta-1}\left(-\frac{T^{2-\beta}}{\beta-2}+\frac{\tau^{2-\beta}}{\beta-2}\right)\right)+\frac{\alpha \tau^{2-\beta}}{2(T-T \beta)}$

$\left.\times\left(2 b\left(1+\frac{1}{\beta-2}\right)+\frac{(\beta-1) \tau H R 2}{\beta-3}\right)-\left(\frac{\mathcal{S P} \vartheta 2}{T}\left(-\frac{\alpha T^{2-\beta}}{\beta-2}-\frac{\alpha T^{1-\beta}(\mathcal{M}-T)}{\beta-1}\right)\right)\right]$

$+2\left[\frac{\mathcal{A}}{T}+\frac{H O 3}{T} \tau \mathrm{W} 1+\frac{H O 3}{T}\left(-\frac{\alpha T^{2-\beta}}{\beta-1}+\frac{\alpha \tau T^{1-\beta}}{\beta-1}+\frac{\alpha}{\beta-1}\left(-\frac{T^{2-\beta}}{\beta-2}+\frac{\tau^{2-\beta}}{\beta-2}\right)\right)\right.$

$+\frac{\alpha \tau^{2-\beta}}{2(T-T \beta)}\left(2 b\left(1+\frac{1}{\beta-2}\right)\left(-\frac{T^{2-\beta}}{\beta-2}+\frac{\tau^{2-\beta}}{\beta-2}\right)\right)+\frac{\alpha \tau^{2-\beta}}{2(T-T \beta)}$

$\left.\times\left(2 b\left(1+\frac{1}{\beta-2}\right)+\frac{(\beta-1) \tau H R 3}{\beta-3}\right)-\left(\frac{\mathcal{S P} \vartheta 3}{T}\left(-\frac{\alpha T^{2-\beta}}{\beta-2}-\frac{\alpha T^{1-\beta}(\mathcal{M}-T)}{\beta-1}\right)\right)\right]$

$+\left[\frac{\mathcal{A}}{T}+\frac{H O 4}{T} \tau \mathrm{W} 1+\frac{H O 4}{T}\left(-\frac{\alpha T^{2-\beta}}{\beta-1}+\frac{\alpha \tau T^{1-\beta}}{\beta-1}+\frac{\alpha}{\beta-1}\right.\right.$

$\left.\times\left(-\frac{T^{2-\beta}}{\beta-2}+\frac{\tau^{2-\beta}}{\beta-2}\right)\right)+\frac{\alpha \tau^{2-\beta}}{2(T-T \beta)}\left(2 b\left(1+\frac{1}{\beta-2}\right)\right.$

$$
\left.\left.\left.+\frac{(\beta-1) \tau H R 4}{\beta-3}\right)-\left(\frac{\mathcal{S P} \vartheta 4}{T}\left(-\frac{\alpha T^{2-\beta}}{\beta-2}-\frac{\alpha T^{1-\beta}(\mathcal{M}-T)}{\beta-1}\right)\right)\right]\right\} .
$$


In the similar lines of the solution procedure for Crisp model, we can obtain the optimal solutions $\tau^{*}, T^{*} \& G M \mathbb{Z}^{*}(\tau)$ for the Fuzzy model.

\section{Numerical examples}

Solutions of the following numerical examples are obtained with the help of Mathematica 11.1.1 software.

Example 1 [Case-1. $\mathcal{M} \leq \tau \leq T]$.

(A) Crisp model.

$$
\begin{aligned}
& \alpha=550, \quad \beta=0.4, \quad \mathcal{A}=1500, \quad \mathcal{H}_{r}=9, \quad \mathcal{H}_{o}=4, \quad \mathrm{~W} 1=225, \\
& \mathcal{P C}=8, \quad \mathcal{S P}=16, \quad \mathcal{M}=0.25, \quad \theta=0.22, \quad \vartheta=0.14
\end{aligned}
$$

Solution:

$$
\begin{aligned}
& \tau=0.484432, \quad T=0.827805, \quad \mathbb{Z} 1=3699.58, \\
& \tau=0.515619, \quad T=0.866313, \quad \mathbb{Z} 2=3347.22, \\
& \tau=0.433552, \quad T=0.764378, \quad \mathbb{Z} 3=3795.11
\end{aligned}
$$

Optimal solution is $\tau^{*}=0.484432, \quad T^{*}=0.827805, \quad \mathbb{Z}^{*}(\tau)=3699.58$.

(B) Fuzzy model.

$$
\begin{aligned}
& \alpha=550, \beta=0.4, \mathcal{A}=1500, \mathcal{H}_{r}=9, \mathcal{H}_{o}=4, \mathrm{~W} 1=225, \\
& \mathcal{P C}=8, \mathcal{S P}=16, \mathcal{M}=0.25, \theta=0.22, \vartheta=0.14, H R 1=5, H R 2=7, \\
& H R 3=9, H R 4=11, H O 1=0, H O 2=2, H O 3=4, H O 4=6, \\
& \theta 1=0.18, \theta 2=0.20, \theta 3=0.22, \theta 4=0.24, \vartheta 1=0.10, \vartheta 2=0.12, \\
& \vartheta 3=0.14 \text { and } \vartheta 4=0.16 .
\end{aligned}
$$

Solution (GMIR method):

$$
\begin{aligned}
& \tau=0.504517, \quad T=0.852635, \quad G M \mathbb{Z} 1=3482.47, \\
& \tau=0.538208, \quad T=0.894049, \quad G M \mathbb{Z} 2=3141.18, \\
& \tau=0.457533, \quad T=0.794371, \quad G M \mathbb{Z} 3=3565.12 .
\end{aligned}
$$

Optimal solution is $\tau^{*}=0.504517, \quad T^{*}=0.852635, \quad G M \mathbb{Z}^{*}(\tau)=3482.47$.

Example 2 [Case 2. $\tau<\mathcal{M} \leq T]$.

\section{(A) Crisp model}

$\alpha=500, \beta=0.2, \mathcal{A}=1630, \mathcal{H}_{r}=12, \mathcal{H}_{o}=5, \mathrm{~W} 1=300, \mathcal{P C}=$ $15, \mathcal{S P}=22, \mathcal{M}=0.35, \theta=0.2$ and $\vartheta=0.12$.

Solution:

$$
\begin{aligned}
& \tau=0.318899, \quad T=0.853285, \quad \mathbb{Z} 1=3789.95, \\
& \tau=0.318864, \quad T=0.853241, \quad \mathbb{Z} 2=3295.97, \\
& \tau=0.302965, \quad T=0.833829, \quad \mathbb{Z} 3=3394.33 .
\end{aligned}
$$


Optimal solution is $\tau^{*}=0.318864, \quad T^{*}=0.853241, \quad \mathbb{Z}^{*}(\tau)=3295.97$.

\section{(B) Fuzzy model.}

$\alpha=500, \beta=0.2, \mathcal{A}=1630, \mathcal{H}_{r}=12, \mathcal{H}_{o}=5, \mathrm{~W} 1=300, \mathcal{P C}=$ $15, \mathcal{S P}=22, \mathcal{M}=0.35, \theta=0.2, \vartheta=0.12, H R 1=8, H R 2=10, H R 3=$ $12, H R 4=14, H O 1=1, H O 2=3, H O 3=5, H O 4=7, \theta 1=0.16, \theta 2=$ $0.18, \theta 3=0.2, \theta 4=0.22, \vartheta 1=0.08, \vartheta 2=0.10, \vartheta 3=0.12$ and $\vartheta 4=0.14$.

Solution (GMIR method):

$$
\begin{aligned}
& \tau=0.337211, \quad T=0.875512, \quad G M \mathbb{Z} 1=3555, \\
& \tau=0.341294, \quad T=0.88045, \quad G M \mathbb{Z} 2=3085.34 \\
& \tau=0.329259, \quad T=0.86586, \quad G M \mathbb{Z} 3=3170.67
\end{aligned}
$$

Optimal solution is $\tau^{*}=0.341294, \quad T^{*}=0.88045, \quad G M \mathbb{Z}^{*}(\tau)=3085.34$.

Example 3 [Case 3. $\tau<T<\mathcal{M}$ ].

(A) Crisp model.

$\alpha=650, \beta=0.3, \mathcal{A}=1500, \mathcal{H}_{r}=9, \mathcal{H}_{o}=4, \mathrm{~W} 1=100, \mathcal{P C}=8, \mathcal{S P}=$ $16, \mathcal{M}=1.2, \theta=0.22$ and $\vartheta=0.14$.

Solution:

$$
\begin{aligned}
& \tau=0.508142, \quad T=0.638222, \quad \mathbb{Z} 1=2760.77, \\
& \tau=0.508228, \quad T=0.638314, \quad \mathbb{Z} 2=2096.29, \\
& \tau=0.423994, \quad T=0.547755, \quad \mathbb{Z} 3=1609.82 .
\end{aligned}
$$

Optimal solution is $\tau^{*}=0.423994, \quad T^{*}=0.547755, \quad \mathbb{Z}^{*}(\tau)=1609.82$.

(B) Fuzzy model.

$\alpha=650, \beta=0.3, \mathcal{A}=1500, \mathcal{H}_{r}=9, \mathcal{H}_{o}=4, \mathrm{~W} 1=100, \mathcal{P C}=8, \mathcal{S P}=$ $16, \mathcal{M}=1.2, \theta=0.22, \vartheta=0.14, H R 1=5, H R 2=7, H R 3=9, H R 4=11$, $H O 1=2, H O 2=3, H O 3=4, H O 4=6, \quad \theta 1=0.18, \quad \theta 2=0.2, \quad \theta 3=0.22$, $\theta 4=0.24, \quad \vartheta 1=0.1, \quad \vartheta 2=0.12, \quad \vartheta 3=0.14$ and $\vartheta 4=0.16$.

Solution (GMIR method):

$$
\begin{aligned}
& \tau=0.528147, \quad T=0.659624, \quad G M \mathbb{Z} 1=2794.2, \\
& \tau=0.530723, \quad T=0.662378, \quad G M \mathbb{Z} 2=2159.56, \\
& \tau=0.443825, \quad T=0.569145, \quad G M \mathbb{Z} 3=1723.33
\end{aligned}
$$

Optimal solution is $\tau^{*}=0.443825, \quad T^{*}=0.569145, \quad G M \mathbb{Z}^{*}(\tau)=1723.33$.

Example 4 [Special case].

(A) Crisp model.

$\alpha=1400, \beta=0.2, \mathcal{A}=2020, \mathcal{H}_{r}=7, \mathcal{H}_{o}=3, \mathrm{~W} 1=300, \mathcal{P C}=6, \mathcal{S P}=$ $11, \mathcal{M}=0.45, \theta=0.3$ and $\vartheta=0.16$.

Solution:

$$
\begin{aligned}
& \tau=0.432606, \quad T=0.620863, \quad \mathbb{Z} 1=5550.04, \\
& \tau=0.459635, \quad T=0.649873, \quad \mathbb{Z} 2=4652.58, \\
& \tau=0.432601, \quad T=0.620857, \quad \mathbb{Z} 3=4760.95
\end{aligned}
$$


Optimal solution is $\tau^{*}=0.459635, \quad T^{*}=0.649873, \quad \mathbb{Z}^{*}(\tau)=4652.58$.

(B) Fuzzy model.

$\alpha=1400, \beta=0.2, \mathcal{A}=2020, \mathcal{H}_{r}=7, \mathcal{H}_{o}=3, \mathrm{~W} 1=300, \mathcal{P C}=6, \mathcal{S P}=$ $11, \mathcal{M}=0.45, \theta=0.3, \vartheta=0.16, H R 1=3, H R 2=5, H R 3=7, H R 4=$ 9, HO1 = 1, HO2 = 2, HO3 = 3, HO4 = 4, $\theta 1=0.26, \quad \theta 2=0.28$, $\theta 3=0.3, \quad \theta 4=0.32, \quad \vartheta 1=0.12, \quad \vartheta 2=0.14, \quad \vartheta 3=0.16$ and $\vartheta 4=0.18$.

Solution (GMIR method):

$$
\begin{aligned}
& \tau=0.446641, \quad T=0.635938, \quad G M \mathbb{Z} 1=5383.18, \\
& \tau=0.476678, \quad T=0.668122, \quad G M \mathbb{Z} 2=4507.03, \\
& \tau=0.450889, \quad T=0.640495, \quad G M \mathbb{Z} 3=4614.53 .
\end{aligned}
$$

Optimal solution is $\tau=0.476678, \quad T=0.668122, \quad G M \mathbb{Z} 2=4507.03$

The following figures depict the inventory level at any time in both the warehouses, consequently the optimal cycle time in both crisp and fuzzy environments for each example discussed.

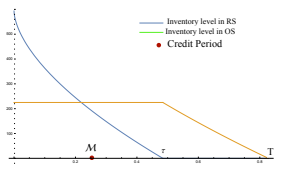

a) Crisp model

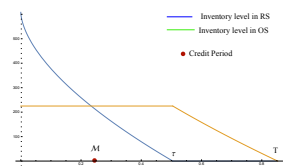

b) Fuzzy model

Figure 2. Inventory model in Example 1.

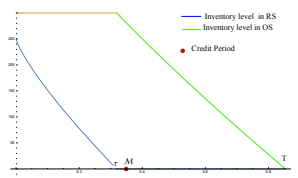

a) Crisp model

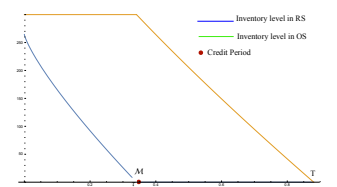

b) Fuzzy model

Figure 3. Inventory model in Example 2.

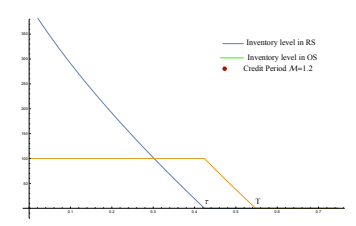

a) Crisp model

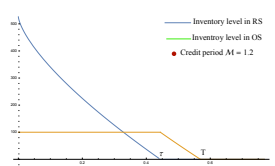

b) Fuzzy model

Figure 4. Inventory model in Example 3. 


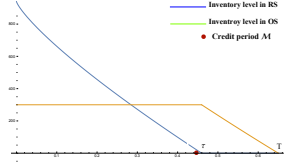

a) Crisp model

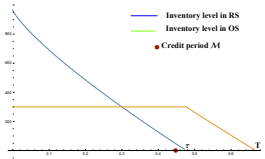

b) Fuzzy model

Figure 5. Inventory model in Example 4.

\section{Sensitivity analysis}

Here we consider the Example 1 to study the sensitivity of different parameters.

On the basis of results shown in Tables 2 and Figures 6-9, it is concluded that:

- The holding cost of OS $\left(\tilde{\mathcal{H}}_{o}\right)$ is very sensitive as the increase in $\tilde{\mathcal{H}}_{o}$ results the increase in total inventory cost; but decrease in vanishing time $(\tau)$ of RS, and so also the total cycle time $T$ (see Table 2 and Figure 6).

- The holding cost of RS $\left(\tilde{\mathcal{H}}_{r}\right)$ is very sensitive as the increase in $\tilde{\mathcal{H}}_{r}$ results the increase in total inventory cost; but decrease in vanishing time $(\tau)$ of RS, and so also the total cycle time $T$ (see Table 2 and Figure 7 ).

- The interest payable $(\tilde{\theta})$ is very sensitive as the increase in $\tilde{\theta}$ results the increase in total inventory cost; but decrease in vanishing time $(\tau)$ of RS, and so also the total cycle time $T$ (see Table 2 and Figure 8 ).

- The interest earned $(\tilde{\vartheta})$ is very sensitive as the increase in $\tilde{\vartheta}$ results the decrease in total inventory cost, vanishing time $(\tau)$ of RS and so also the total cycle time $T$ (see Table 2 and Figure 9 ).

Table 2. Sensitivity of different cost parameters.

\begin{tabular}{|c|c|c|c|c|c|c|c|}
\hline \multicolumn{4}{|c|}{ Sensitivity of holding cost parameter $\tilde{\mathcal{H}}_{o}$. } & \multicolumn{4}{|c|}{ Sensitivity of holding cost parameter $\tilde{\mathcal{H}}_{r}$. } \\
\hline $\mathcal{H}_{o}$ & $\tau$ & $T$ & $G M \mathbb{Z}(\tau)$ & $\overline{\mathcal{H}}_{r}$ & $\tau$ & $T$ & $G M \mathbb{Z}(\tau)$ \\
\hline 4,6$)$ & 517 & 835 & & 11) & 504517 & 2635 & 3482.47 \\
\hline & 603 & & & & & & \\
\hline & 89 & 0. & & & 23 & & .66 \\
\hline & & & & & 218 & & .21 \\
\hline$(4,6,8,10)$ & 0.484461 & 0.827841 & 4190.78 & $(13,15,17,19)$ & 0.442472 & 0.775557 & 3629.83 \\
\hline
\end{tabular}

\begin{tabular}{|c|c|c|c|c|}
\hline \multicolumn{2}{|l|}{ Sensitivity of parameter $\tilde{\theta}$. } & \multicolumn{3}{|c|}{ Sensitivity of parameter $\tilde{\vartheta}$. } \\
\hline$\tilde{\theta}$ & $\overline{T G M \mathbb{Z}(\tau)}$ & $\tilde{\vartheta}$ & $T$ & $G M \mathbb{Z}(\tau)$ \\
\hline$(.18, .20, .22, .24) 0.5045170 .852635$ & $5 \quad 3482.47$ & $(.10, .12, .14, .16)$ & 0.5045170 .852635 & 3482.47 \\
\hline $2, .24, .26) 0.495458 \quad 0.84145$ & $5 \quad 3536.88$ & $4, .16, .18)$ & 9364 & 3468.4 \\
\hline$(.22, .24, .26, .28) 0.4$ & $9 \quad 3590.67$ & $6, .18$, & 6079 & 3454.28 \\
\hline$(.24, .26, .28, .30) 0.478452 \quad 0.82039$ & $9 \quad 3643.86$ & $18, .20, .22)$ & 0.4965330 .842778 & 3440.1 \\
\hline$(.26, .28, .30, .32) 0.4704660 .810473$ & $3 \quad 3696.46$ & $(.18, .20, .22, .24)$ & 0.4938510 .839463 & 3425.87 \\
\hline
\end{tabular}




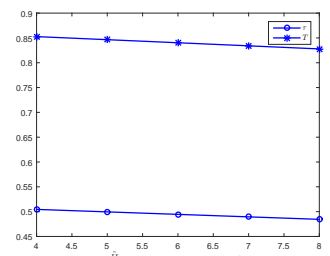

a) $\tilde{\mathcal{H}}_{o}$ vs $\tau$ and $T$

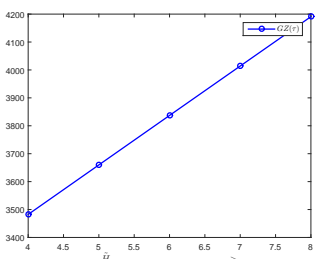

b) $\tilde{\mathcal{H}}_{o}$ vs $G M \mathbb{Z}(\tau)$

Figure 6. Sensitivity effect of parameter $\tilde{\mathcal{H}}_{o}$ on Inventory.

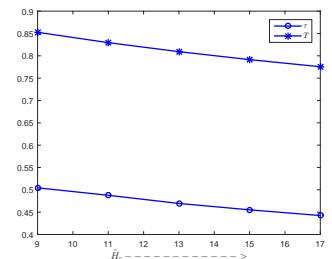

a) $\tilde{\mathcal{H}}_{r}$ vs $\tau$ and $T$

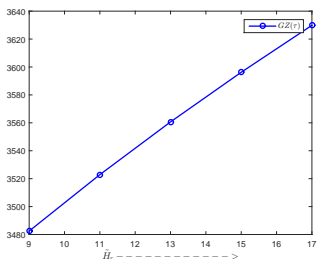

b) $\tilde{\mathcal{H}}_{r}$ vs $G M \mathbb{Z}(\tau)$

Figure 7. Sensitivity effect of parameter $\tilde{\mathcal{H}}_{r}$ on Inventory.

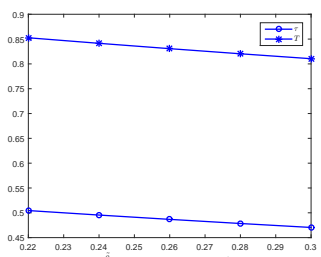

a) $\tilde{\theta}$ vs $\tau$ and $T$

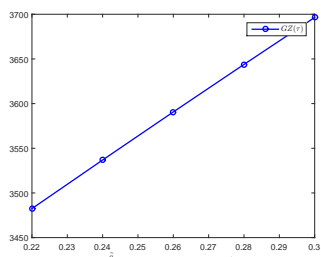

b) $\tilde{\theta}$ vs $G M \mathbb{Z}(\tau)$

Figure 8. Sensitivity effect of parameter $\tilde{\theta}$ on Inventory.

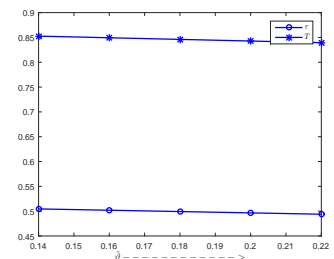

a) $\tilde{\vartheta}$ vs $\tau$ and $T$

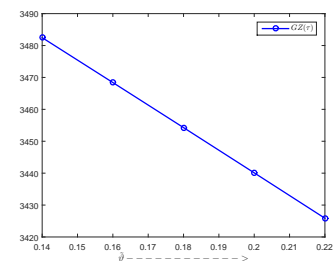

b) $\tilde{\vartheta}$ vs $G M \mathbb{Z}(\tau)$

Figure 9. Sensitivity effect of parameter $\tilde{\vartheta}$ on Inventory.

\section{Conclusions}

Inventory control of products having two-storage facility under acceptable delay in payment is quite relevant in many business organizations. In this paper, a two storage inventory model under acceptable delay in payment for items having life time more than that of the cycle time with decreasing time dependent 
demand, variable holding cost (in RS) is developed successfully in both crisp and fuzzy environment. It has been observed from the mentioned examples that, the optimal results in fuzzy environment differ from the crisp environment. That is, the imprecision of costs lead the total inventory cost to excess or low. As the fact, the managers of inventory always look for accurate results to minimize the inventory cost. So, we suggest that the fuzzy inventory model is best suitable for the real world inventory problems. Also, the sensitivity of the parameters shows that the optimal replenishment time and cost depends on the cost of those parameters. The corresponding figures (Figure 6-Figure 9) and table (Table 2) of sensitivity of parameters draw the visible attention justifying our arguments. Thus, the sensitivity analysis section helps the inventory managers in decision making.

\section{Future Research Directions}

The present model can be extended in several directions. One is shortage with different backlogging can be incorporated. Second one is different demands and deterioration can be considered. Third one is the model can be considered for imperfect production inventory of deteriorating items. Fourth one is the trade credit period can be linked to ordered quantity. Furthermore, the model can be extended in different direction by considering various inventory constraints.

\section{References}

[1] H.K. Alfares. Maximum-production inventory model with stockdependent demand, time-dependent holding cost, and all-units quantity discounts. Math. Model. Anal., 20(6):715-736, 2015. https://doi.org/10.3846/13926292.2015.1108936.

[2] S. Barik, S.K. Paikray, S. Mishra, B.A. Kumar and U.K. Misra. Optimal inventory model with single item under various demand conditions. Int. J. Adv. Res. Sci. Eng., 6:1577-1588, 2017.

[3] S. Barik, S.K. Paikray, S. Mishra and U.K. Misra. Inventory models for deteriorating items for non linear holding cost with time dependant demand. J. Adv. Math., 9:2705-2709, 2014.

[4] A.K. Bhunia, C.K. Jaggi, A. Sharma and R. Sharma. A two-warehouse inventory model for deteriorating items under permissible delay in payment with partial backlogging. Appl. Math. Comput., 232(1):1125-1137, 2014. https://doi.org/10.1016/j.amc.2014.01.115.

[5] D. Chakraborty, D.K. Jana and T.K. Roy. Two-warehouse partial backlogging inventory model with ramp type demand rate, three-parameter Weibull distribution deterioration under inflation and permissible delay in payments. Comput. Ind. Eng., 123:157-159, 2018. https://doi.org/10.1016/j.cie.2018.06.022.

[6] P. Guchhait, M.K. Maiti and M. Maiti. Two storage inventory model with fuzzy deterioration over a random planning horizon. Appl. Soft Comput., 13(1):428448, 2013. https://doi.org/10.1016/j.asoc.2012.07.028.

[7] C.W. Haley and H.K. Higgins. Inventory policy and trade credit financing. Manag. Sci., 20(4):464-471, 1973. https://doi.org/10.1287/mnsc.20.4.464. 
[8] R.V. Hartley. Operations Research-A Managerial Emphasis. Good Year Publishing Company, California, 1976.

[9] S. K. Indrajitsingha, P. N. Samanta and U.K. Misra. A fuzzy two-warehouse inventory model for single deteriorating item with selling-price-dependent demand and shortage under partial-backlogged condition. Appl. Appl. Math., 14:511-536, 2019 .

[10] C.K. Jaggi, L.E. Cárdenas-Barrón, S. Tiwari and A.A. Shafi. Two-warehouse inventory model for deteriorating items with imperfect quality under the conditions of permissible delay in payments. Sci. Iran. E., 24(1):390-412, 2017. https://doi.org/10.24200/sci.2017.4042.

[11] C.K. Jaggi, P. Gautam and A. Khanna. Inventory decisions for imperfect quality deteriorating items with exponential declining demand under trade credit and partially backlogged shortages. In P. Kapur, U. Kumar and A. Verma(Eds.), Quality, IT and Business Operations., Springer Proceedings in Business and Economics, pp. 213-229, Singapore, 2018.

[12] C.K. Jaggi, S. Pareek, A. Khanna and R. Sharma. Credit financing in a twowarehouse environment for deteriorating items with price-sensitive demand and fully backlogged shortages. Appl. Math. Model., 38(21-22):5315-5333, 2014. https://doi.org/10.1016/j.apm.2014.04.025.

[13] C.K. Jaggi, S. Tiwari and S.K. Goel. Credit financing in economic ordering policies for non-instantaneous deteriorating items with price dependent demand and two storage facilities. Ann. Oper. Res., 248:253-280, 2017. https://doi.org/10.1007/s10479-016-2179-3.

[14] N.K. Kaliraman, R. Raj, S. Chandra and H. Chaudhary. Two warehouse inventory model for deteriorating item with exponential demand rate and permissible delay in payment. Yugosl. J. Oper. Res., 27(1):109-124, 2017. https://doi.org/10.2298/YJOR150404007K.

[15] A. Khanna, M. Mittal P. Goutam and C.K. Jaggi. Credit financing for deteriorating imperfect quality items with allowable shortages. Decis. Sci. Lett., 5:45-60, 2016. https://doi.org/10.5267/j.dsl.2015.9.001.

[16] A. Khanna, P. Goutam and C.K. Jaggi. Inventory modeling for deteriorating imperfect quality items with selling price dependent demand and shortage backordering under credit financing. Int. J. Math. Eng. Manag. Sci., 2(2):110-124, 2017. https://doi.org/10.33889/IJMEMS.2017.2.2-010.

[17] D. Khurana. Two warehouse inventory model for deteriorating items with time dependent demand under inflation. Int. J. Comput. Appl., 114(7):34-38, 2015. https://doi.org/10.5120/19994-1738.

[18] Y. Liang and F. Zhou. A two-warehouse inventory model for deteriorating items under conditionally permissible delay in payment. Appl. Math. Model., 35(5):2221-2231, 2011. https://doi.org/10.1016/j.apm.2010.11.014.

[19] J-J. Liao, K-J. Chung and K-N. Huang. A deterministic inventory model for deteriorating items with two warehouses and trade credit in a supply chain system. Int. J. Prod. Econ., 146(2):557-565, 2013. https://doi.org/10.1016/j.ijpe.2013.08.001.

[20] J-J. Liao, K-N. Huang and K-J. Chung. Lot-sizing decisions for deteriorating items with two warehouses under an order-size-dependent trade credit. Int. J. Prod. Econ., 137(1):102-115, 2012. https://doi.org/10.1016/j.ijpe.2012.01.020. 
[21] N. Misra and S. Mishra. Fuzzy inventory model with single item under constant demand and time dependent holding cost. J. progre. Res. Math., 7:949-961, 2016.

[22] R.S. Rajan and R. Uthayakumar. A two-warehouse inventory model for deteriorating items with permissible delay under exponentially increasing demand. Int. J. Suppl. Oper. Manag., 2:662-682, 2015.

[23] S.S. Routray, S.K. Paikray, S. Misra and U.K. Misra. Fuzzy inventory model with single item under time dependent demand and holding cost. Int. J. Adv. Res. Sci. Eng., 6:1604-1618, 2017.

[24] B.K. Sett, B. Sarkar and A. Goswami. A two- warehouse inventory model with increasing demand and time varying deterioration. Sci. Iran., 19:1969-1977, 2012. https://doi.org/10.1016/j.scient.2012.10.040.

[25] S. Shabani, A. Mirzazadeh and E. Sharifi. A two-warehouse inventory model with fuzzy deterioration rate and fuzzy demand rate under conditionally permissible delay in payment. J. Ind. Prod. Eng., 33(2):134-142, 2016. https://doi.org/10.1080/21681015.2015.1107859.

[26] D. Singh and A. Kumar. Two-warehouses partial backlogging inventory model for deteriorating items with selling price dependent demand under trade credit in the inflationary environment. Int. J. Pure Appl. Math., 118:1447-1457, 2018. https://doi.org/10.1504/IJOR.2011.040329.

[27] S.R. Singh, N. Kumar and R. Kumari. Two-warehouse fuzzy inventory model under the conditions of permissible delay in payments. Int. J. Oper. Res., 11(1):7899, 2011.

[28] S. Tiwari, L.E. Crdenas-Barrn, A. Khanna and C.K. Jaggi. Impact of trade credit and inflation on retailer's ordering policies for non-instantaneous deteriorating items in a two-warehouse environment. Int. J. Prod. Econ., 176:154-169, 2016.

[29] H-L. Yang. Two-warehouse partial backlogging inventory models with threeparameter weibull distribution deterioration under inflation. Int. J.Prod. Econ., 138(1):107-116, 2012. https://doi.org/10.1016/j.ijpe.2012.03.007.

[30] J.C.P. Yu. Optimizing a two-warehouse system under shortage backordering, trade credit, and decreasing rental conditions. Int. J.Prod. Econ., 209:147-155, 2019. https://doi.org/10.1016/j.ijpe.2018.06.003. 